\title{
Correlation of olfactory dysfunction with lung involvement and severity of COVID-19
}

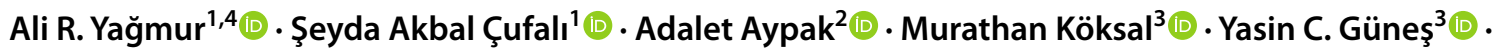 \\ Kürşat M. Özcan ${ }^{1}$ (1)
}

Received: 27 June 2021 / Accepted: 29 July 2021 / Published online: 10 August 2021

(c) Royal Academy of Medicine in Ireland 2021

\begin{abstract}
Background Olfactory dysfunction (OD) is a significant symptom of COVID-19 and may be the earliest symptom, or it may sometimes be the only manifestation of the disease.

Aims To investigate whether OD is correlated with chest computed tomography (CT) findings, blood test parameters, and disease severity in COVID-19 patients.

Methods The files of COVID-19 patients were retrospectively reviewed, and the ones who had information about smelling status and CT were taken into consideration. A total of 180 patients were divided into two groups: the OD group consisted of 89 patients with self-reported OD, and the No-OD group consisted of 91 subjects who did not complain of OD. The two groups were compared for the amount of lung consolidation on CT, intensive care unit (ICU) admission, and blood test parameters (complete blood count, alanine aminotransferase (ALT), aspartate aminotransferase (AST), creatine kinase (CK), lactate dehydrogenase (LDH), C-reactive protein (CRP), erythrocyte sedimentation rate (ESR), ferritin, D-dimer, interleukin-6 (IL-6)).

Results The amount of lung consolidation and ICU admission were significantly higher in the No-OD group $(p<0.001$ for both). White blood cell $(p=0.06)$, monocyte $(p=0.26)$, and platelet $(p=0.13)$ counts and hemoglobin $(p=0.63)$, ALT $(p=0.89)$, and D-dimer $(p=0.45)$ levels of the two groups were similar. Lymphocyte count $(p=0.01)$, neutrophil count $(p=0.01)$, and AST $(p=0.03), \operatorname{CK}(p=0.01), \operatorname{LDH}(p<0.001), \operatorname{CRP}(p<0.001)$, ESR $(p<0.001)$, ferritin $(p<0.001)$, and IL-6 $(p<0.001)$ levels were significantly higher in the No-OD group.

Conclusions The patients presenting to the hospital with self-reported OD may have less lung involvement and a milder disease course compared to patients without OD on admission.
\end{abstract}

Keywords Anosmia $\cdot$ Chest computed tomography $\cdot$ COVID-19 Intensive care units · Olfactory dysfunction · SARSCoV-2

\section{Introduction}

Coronavirus disease of 2019 (COVID-19), caused by severe acute respiratory syndrome coronavirus 2 (SARSCoV-2), was first reported in Wuhan City of China in
2019 and was declared as a global pandemic by the World Health Organization on March 11, 2020. Its frequent symptoms are dry cough, fever, fatigue, myalgia, arthralgia, headache, sore throat, nasal obstruction, diarrhea, and

\section{Kürşat M. Özcan}

kursat.murat@gmail.com

1 Department of Otorhinolaryngology, Head and Neck Surgery, Ankara City Hospital, Ankara, Turkey

2 Department of Clinical Microbiology and Infectious Diseases, Ankara City Hospital, Ankara, Turkey

3 Department of Radiology, Ankara City Hospital, Ankara, Turkey

4 Ankara, Turkey 
olfactory dysfunction (OD). However, some infected individuals may be asymptomatic.

SARS-CoV-2 may infect both the upper and lower airways. It causes mild cold-like symptoms in the upper respiratory tract; however, it may lead to severe dyspnea when the lungs are involved. Chest CT may help the diagnosis of COVID-19 since it has a high sensitivity for its pulmonary involvement and may be performed when polymerase chain reaction (PCR) for SARS-CoV-2 is negative, but there is a high clinical suspicion for COVID-19 [1]. C-reactive protein (CRP), interleukin-6 (IL-6), erythrocyte sedimentation rate, serum amyloid A, ferritin, urea, creatinine, and lactate dehydrogenase (LDH) levels have been shown to be elevated in severe COVID-19 [2]. Both thorax CT and laboratory parameters are valuable tools for both the diagnosis and monitoring of hospitalized patients.

OD is a significant symptom of COVID-19. In fact, OD may appear following viral upper respiratory tract (URT) infections caused by rhinovirus, influenza virus, parainfluenza virus, adenovirus, and respiratory syncytial virus $[3,4]$. The American Academy of Otolaryngology-Head and Neck Surgery included anosmia among the symptoms of COVID-19 on March 22, 2020 [5]. Various authors reported a higher incidence of loss of smell in COVID-19 compared to other viral URT infections, and some others suggested that smell and taste dysfunction might be correlated with the severity of the infection [6-8].

The aim of the present study is to investigate whether self-reported OD is correlated with chest CT findings, blood test parameters, and disease severity, represented as intensive care unit (ICU) admission, in patients with COVID-19. To our knowledge, this is the first study in the literature that compares the amount of lung consolidation between the subjects with and without OD.

\section{Material and methods}

This study was conducted in a tertiary care pandemic hospital, after approval of the study protocol by Institutional Ethics Committee (decree no. E1-20-1346).

The medical records of 180 patients aged between 18 and 65 years, diagnosed with COVID-19 based on RTPCR (real-time reverse transcriptase polymerase chain reaction), and treated in our hospital between April 2020 and June 2020 were retrospectively reviewed including ICU admission, using the hospital's electronic patient recording system.

The symptoms and the physical examination findings on hospital admission were reviewed. The patients who did not have concomitant nasal obstruction or rhinorrhea and had sudden-onset OD and chest CTs were included in the study.
Exclusion criteria were smoking, presence of diabetes mellitus, asthma, allergic rhinitis, nasal polyposis, a previous history of taste and smell impairment, and previous use of supplemental oxygen therapy.

The patients were divided into two groups as the patients with self-reported OD and the ones that did not have this complaint. Of 144 patients with self-reported OD, 47 smokers, 11 patients with diabetes mellitus, 5 patients with asthma, and 7 patients with allergic rhinitis and/or nasal polyposis were excluded. Finally, 89 COVID-19-positive patients with OD were included in the study group (OD group). The No-OD group consisted of 91 non-smoking, COVID-19-positive patients that did not have loss of smell or any systemic disorders. These patients were randomly selected among the COVID-19-positive patients who underwent chest $\mathrm{CT}$ imaging.

All patients included in the study had their thorax CTs in our hospital. The same radiologist re-examined all CT scans. CT scans were obtained while the patient was in supine position and at the end-inspiratory phase, using the 128-slice Revolution (GE Healthcare, IL, USA) CT device. Scanning parameters of the CT device were as follows: beam collimation, $1.3 \mathrm{~mm}$; interval, $2.5 \mathrm{~mm}$; tube voltage, $100-120 \mathrm{kV}$; mA s (tube current $\times$ time), 130-200; tube current, $240 \mathrm{~mA}$; and pitch, 1.4. The slice thickness after reformatting was $2.5 \mathrm{~mm}$. Non-contrast CT scans of the patients were transferred to the AW VolumeShare 7 workstation with the Thoracic VCAR software in order to quantify lung involvement. The total lung volume and the percentage of lung volume occupied by consolidation were calculated for both lungs [9]. Poor-quality images due to patient movement, artifacts, or technical errors not allowing measurements were excluded from the analysis. The percentages of lung volumes occupied by consolidation areas were compared between the two study groups.

White blood cell (WBC), neutrophil, lymphocyte, monocyte, and platelet (PLT) counts and hemoglobin (HGB), alanine aminotransferase (ALT), aspartate aminotransferase (AST), creatine kinase (CK), LDH, CRP, erythrocyte sedimentation rate (ESR), ferritin, D-dimer, and IL-6 levels obtained from peripheral blood samples were compared between the OD and No-OD groups. ADVIA 2120 Hematology System (Siemens Healthcare Diagnostics, Erlangen, Germany) was used for complete blood count analysis, and biochemical parameters were studied using Atellica Solution Immunoassay \& Clinical Chemistry Analyzer (Siemens Healthcare Diagnostics, Erlangen, Germany) and D-Dimer Sysmex CS-5100 System (Siemens Healthcare Diagnostics, Erlangen, Germany).

\section{Statistical analysis}

The MATLAB (matrix laboratory) statistics toolbox (2020b) was used for all statistical analyses. 
One-sample Kolmogorov-Smirnov test was used to test the normality of the distribution of the percentages of lung volumes with consolidation on chest CT, and the blood test parameters, in the total sample and two study groups. Then, two-sample Kolmogorov-Smirnov test was employed to determine whether the two groups had significant differences in terms of age, blood parameters, or the percentages of lung volumes with consolidation. Since the two study groups showed differences for the age and gender distributions, the effects of age and/or gender were simultaneously analyzed with multivariate linear regression analysis.

Multivariate logistic regression analysis was conducted to determine the risk factors for ICU admission. Variables with a $p$ value of $<0.2$ in the univariate analysis were included in the multivariate logistic regression models. A backward elimination (likelihood ratio) modeling strategy was used. The odds ratios (ORs) along with their corresponding 95\% confidence intervals (CIs) were presented for the effect size of each of the risk factors.

Normally distributed continuous variables were expressed as mean \pm standard deviation, and non-normally distributed variables were presented as median and minimum-maximum. Categorical variables were presented as numbers. A $p$-value $<0.05$ was considered statistically significant.

\section{Results}

Among the 180 patients included in the study, self-reported OD was present in 89 patients (OD group) and absent in 91 patients (No-OD group). There were $33(37 \%)$ males and $56(63 \%)$ females in the OD group and $36(40 \%)$ males and $55(60 \%)$ females in the No-OD group. The mean age was $40.7 \pm 14.3$ years in the OD group and $46.7 \pm 11.06$ years in the No-OD group. Age and gender distributions were significantly different in two study groups $(p=0.001)$.

There were no significant differences between the OD and No-OD groups for WBC, PLT, monocyte counts, HGB, ALT, D-dimer levels ( $p=0.06, p=0.26, p=0.63, p=0.13$, $p=0.89, p=0.45$, respectively). Neutrophil counts and AST, CK, LDH, CRP, ESR, ferritin, and IL-6 levels were significantly higher; however, lymphocyte counts were significantly lower in the No-OD group $(p=0.01, p=0.01$, $p=0.01, p<0.001, p<0.001, p<0.001, p<0.001, p<0.001$, and $p=0.03$, respectively) (Table 1 ).

The lung consolidation area percentage, determined as the percentage of the lung volume occupied by consolidation areas in the right lung, left lung, and bilaterally, was compared between the groups. The percentage of lung volume with consolidation (right, left, bilateral) was significantly smaller in the OD group compared to that in the No-OD group ( $p<0.001$ for all) (Table 2).
Table 1 Comparisons of blood parameter levels between OD and No-OD groups

\begin{tabular}{llllr}
\hline & Normal range & OD group & No-OD group & $p$ \\
\hline WBC $\left(\times 10^{9} / \mathrm{L}\right)$ & $3.9-10.2$ & $4.8 \pm 2.4$ & $6.1 \pm 2.2$ & 0.06 \\
Neutrophils $\left(\times 10^{9} / \mathrm{L}\right)$ & $1.5-7.7$ & $3.1 \pm 1.8$ & $4.3 \pm 2.1$ & 0.01 \\
Lymphocytes $\left(\times 10^{9} / \mathrm{L}\right)$ & $1.1-4.5$ & $1.2 \pm 0.7$ & $1.1 \pm 0.4$ & 0.01 \\
Monocytes $\left(\times 10^{9} / \mathrm{L}\right)$ & $2-9.5$ & $0.3 \pm 0.2$ & $0.3 \pm 0.1$ & 0.26 \\
HGB $(\mathrm{g} / \mathrm{dL})$ & Male $13.5-17.2$ & $13.7 \pm 1.9$ & $13.8 \pm 1.7$ & 0.63 \\
& Female $12-15.6$ & & & \\
PLT $\left(\times 10^{9} / \mathrm{L}\right)$ & $150-400$ & $214 \pm 106.3$ & $223 \pm 77.6$ & 0.13 \\
ALT $(\mathrm{U} / \mathrm{L})$ & $<50$ & $27(7-130)$ & $27(5-653)$ & 0.89 \\
AST $(\mathrm{U} / \mathrm{L})$ & $<35$ & $24(11-74)$ & $29(10-288)$ & 0.03 \\
LDH $(\mathrm{U} / \mathrm{L})$ & $120-246$ & $206(98-538)$ & $279(19-1224)$ & $<0.001$ \\
CK $(\mathrm{U} / \mathrm{L})$ & $32-294$ & $74(22-494)$ & $126(21-9100)$ & 0.01 \\
CRP $(\mathrm{mg} / \mathrm{L})$ & $0-5$ & $4(0-119)$ & $38(2-251)$ & $<0.001$ \\
ESR $(\mathrm{mm} / \mathrm{h})$ & $0-20$ & $15.5(1-385)$ & $32(3-789)$ & $<0.001$ \\
D-dimer $(\mathrm{mg} / \mathrm{L})$ & $<0.55$ & $0.5(0.2-2.8)$ & $0.6(0.2-11.3)$ & 0.45 \\
Ferritin $(\mu \mathrm{\mu g} / \mathrm{L})$ & $22-322$ & $74.5(2-1079)$ & $250(6-1805)$ & $<0.001$ \\
IL-6 $(\mathrm{pg} / \mathrm{mL})$ & $0-3.4$ & $6.2(2.6-79.9)$ & $14.6(2-103)$ & $<0.001$ \\
\hline
\end{tabular}

The values are presented as mean $\pm \mathrm{SD}$, or median (minimum-maximum)

$O D$ olfactory dysfunction, $W B C$ white blood cell, $H G B$ hemoglobin, $P L T$ platelet, $A L T$ alanine aminotransferase, $A S T$ aspartate aminotransferase, $C K$ creatine kinase, $L D H$ lactate dehydrogenase, $C R P$ C-reactive protein, $E S R$ erythrocyte sedimentation rate, $I L-6$ interleukin-6 
Since age and gender distribution were significantly different between two study groups, we investigated whether these two parameters had any effect on other parameters studied. The statistical analysis showed that advanced age had a statistically significant positive effect on lung involvement $(p<0.001)$, but gender did not $(p=0.69)$. The multivariate linear regression analysis performed to eliminate the effect of age showed that OD was an independent significant negative factor for lung involvement (OR $-3.13,95 \%$ CI -5.49 to $-0.77, p=0.01$ ).

When all patients included in the study are considered, a total of 33 patients, [10] (13.3\%) females and 23 (26.1\%) males needed ICU admission. There was no difference between the genders for ICU admission $(p=0.043)$. The mean age of the patients who needed ICU care was significantly higher $(49.73 \pm 12.89$ vs. $42.1 \pm 12, p=0.002)$. When the OD was taken into consideration, $5(6.9 \%)$ patients (1 female and 4 males, mean age $48.50 \pm 14.22$ ) with OD and $28(30.8 \%)$ patients ( 9 females and 19 males, mean age $49.50 \pm 12.49$ ) without OD needed ICU admission, with a significant difference between the OD and No-OD groups $(p<0.001)$. Multivariate regression analysis showed that the "absence" of OD (OR 4.83, 95\% CI 1.72-13.5, $p=0.003$ ) and older age (OR 2.68, 95\% CI 1.17-6.17, $p=0.02$ ) were risk factors for ICU. We could not perform any statistical analysis for age or gender differences between patients needing ICU in OD and No-OD groups, since the number of patients in the OD group was small (4 patients).

\section{Discussion}

In this study, we investigated whether the presence of OD was correlated with chest CT findings, blood test parameters, and disease severity, represented as intensive care unit (ICU) admission in patients with COVID-19, and found that the patients with self-reported OD had significantly less lung involvement and less need for ICU.

The clinical course of COVID-19 is variable and ranges between asymptomatic infection and acute respiratory distress syndrome (ARDS) [11]. In severe cases, dyspnea and/ or hypoxia may be seen due to lung involvement and the

Table 2 Comparison of percentages of lung volumes occupied by consolidation between OD and No-OD groups

\begin{tabular}{llll}
\hline & \multicolumn{2}{l}{ Percentage of consolidation } & $p$ \\
\cline { 2 - 3 } & OD group & No-OD group & \\
\hline Right lung & $4.4(0-38.5)$ & $9.5(0-49.8)$ & $<0.001$ \\
Left lung & $4.4(0-53.8)$ & $8.3(0-46)$ & $<0.001$ \\
Bilateral & $4.3(0-50.9)$ & $9.2(0-48.4)$ & $<0.001$ \\
\hline
\end{tabular}

Values are presented as median (minimum-maximum) patient's condition may rapidly deteriorate and progress to ARDS [11]. However, it is not easy to predict which patient will progress into the severe form.

Olfactory and gustatory disorders have been frequently reported in COVID-19 patients [12-15]. Chemosensitive dysfunction may be the earliest symptom, or it may sometimes be the only manifestation of the disease. Unlike the loss of smell that occurs in other viral upper respiratory tract infections, anosmia associated with COVID-19 may occur without nasal obstruction [16]. Loss of smell and taste may also be used as an indicator of potential infection with SARS-CoV-2 [17]. In our hospital, the patients with suspected COVID-19 were questioned about loss of smell, and this information was recorded in their files.

A meta-analysis that included 19,424 COVID-19 patients from 27 articles reported pooled prevalence of loss of smell as $48.47 \%$ in COVID-19 patients (1). Various authors investigated the correlation of OD with severity of COVID-19, or its pulmonary symptoms, and reported conflicting results. Speth et al. made a survey study on 103 COVID-19 patients with OD and reported that 22 (21.3\%) of them were hospitalized, and the patients with OD experienced generally more severe shortness of breath compared to patients that did not experience OD [18]. Vaira et al. included healthcare workers with OD in their study and reported that duration of chemosensitive symptoms greater than 7 days showed a statistically significant correlation with the development of moderate and severe COVID-19 [19]. On the other hand, Haehner et al. studied 22 PCR-positive COVID-19 patients with sudden smell and taste loss and reported that COVID-19 patients with olfactory loss had less severe symptoms [20]. None of the aforementioned studies examined the correlation of chest CT findings with OD. In our study, we examined the thorax CTs of the patients with and without OD and found that the patients with OD had less severe lung involvement $(p<0.001)$, and they needed ICU admission less $(p<0.001)$ compared to the patients who did not have OD.

In our study, we employed ICU admission, not hospitalization, as an indicator of the disease severity, beca Sanli et al. investigated the relationship betweenuse at the time we collected data, the number of COVID-19 cases was relatively small in our country, and all COVID-19 patients were being hospitalized for isolation purposes, regardless of their clinical condition. In addition, nearly all patients admitting to our hospital with COVID-19 symptoms were ordered chest $\mathrm{CT}$ on their admission, in line with our hospital's policy.

It has been reported that the extent of pulmonary involvement on chest $\mathrm{CT}$ is associated with the prognosis of COVID-19, in addition to demographic factors and comorbidities [21-23]. Francone et al. reported that chest CT scores were highly correlated with disease severity and laboratory findings, and that $\mathrm{CT}$ imaging could be useful 
for rapid diagnosis in symptomatic cases [24]. In our study, we compared lung involvement between patients with and without OD and found the percentage of lung volume with consolidation (right, left, bilateral) significantly smaller in patients with OD $(p<0.001)$. In correlation with this finding, our patients with OD needed ICU admission less $(p<0.001)$ compared to the patients who did not have OD.

It has been suggested that various serum biomarkers might serve as potential indicators for severe disease [25]. In ICU patients with the patients hospitalized in the general ward, Huang et al. found higher WBC and neutrophil counts and ALT, LDH, and D-dimer levels, but lower lymphocyte counts [26]. Sanli et al. investigated the relationship between IL-6 levels and the presence of anosmia in COVID19 patients; although IL-6 levels were above normal limits in both patients with and patients without anosmia, the patients with anosmia had lower IL-6 levels [27]. In our study, we detected lower LDH, ESR, CRP, ferritin, and IL-6 levels in patients with OD, indicating less severe disease.

Chen et al. reported lower lymphocyte counts in patients with severe COVID-19 symptoms compared to those in patients with mild symptoms [28]. In another study on COVID-19 patients, Foster et al. found that lymphopenia was less common, but a higher albumin level was more common in patients with smell loss compared to patients without smell loss, but other laboratory parameters and inflammatory markers were not associated with loss of smell [29]. In our study, WBC, monocyte, and PLT counts and HGB and ALT levels were not significantly different between the two study groups; however, higher lymphocyte counts, lower neutrophil counts, and lower AST levels were observed in the group with OD.

High D-dimer levels were claimed to predict mortality in COVID-19 patients, and the patients who died were also found to have higher CRP and LDH values compared to patients who survived [30]. Some studies showed that almost all hospitalized COVID-19 patients had elevated LDH and $\mathrm{CK}$ values [26, 31, 32]. We did not find any significant difference between OD and No-OD groups for D-dimer levels; however, LDH and CK levels were higher in the No-OD group.

\section{Limitations of the study}

Lack of pre- and post-COVID-19 objective smell tests and inclusion of the patients with self-reported, sudden-onset OD are the limitations of our study.

\section{Conclusion}

In conclusion, OD is one of the symptoms of COVID-19 patients, and our results indicate that the patients presenting to the hospital with self-reported OD may have less lung involvement and a milder disease course compared to patients without OD on admission. At times when the pandemic strikes hard and hospitals are overwhelmed, this piece of information can be useful in the triage of patients. There is a need for further large-scale studies confirming OD with smell tests, closely monitoring the clinical course, laboratory data, and radiological imaging of the patients with COVID-19.

\section{Declarations}

Ethical approval was obtained from the institutional review board prior to initiation of the study (Ankara City Hospital, No. 1 clinical research ethics committee, decree no. E1-20-1346).

Conflict of interest The authors declare no competing interests.

\section{References}

1. Lv M, Wang M, Yang N et al (2020) Chest computed tomography for the diagnosis of patients with coronavirus disease 2019 (COVID-19): a rapid review and meta-analysis. Ann Transl Med $8: 622$

2. Zhang L, Guo H (2020) Biomarkers of COVID-19 and technologies to combat SARSCoV-2. Advances in Biomarker Sciences and Technology 2:1-23

3. Allis TJ, Leopold DA (2012) Smell and taste disorders. Facial Plast Surg Clin North Am 20:93-111

4. Suzuki M, Saito K, Min WP et al (2007) Identification of viruses in patients with postviral olfactory dysfunction. Laryngoscope 117:272-277

5. Anosmia, hyposmia, and dysgeusia symptoms of coronavirus disease (2020) American Academy Otolaryngology - Head and Neck Surgery. https://www.entnet.org/content/aao-hns-anosmiahyposmia-and-dysgeusia-symptoms-coronavirus-disease. Accessed 5 April 2020

6. Ibekwe TS, Fasunla AJ, Orimadegun AE (2020) Systematic review and meta-analysis of smell and taste disorders in COVID-19. OTO Open 4:2473974X20957975

7. Printza A, Constantinidis J (2020) The role of self-reported smell and taste disorders in suspected COVID-19. Eur Arch Otorhinolaryngol 277:2625-2630

8. Mazzatenta A, Neri G, D'Ardes D et al (2020) Smell and taste in severe CoViD-19: self-reported vs. testing. Front Med (Lausanne) 7:589409

9. Grassi R, Cappabianca S, Urraro F et al (2020) Chest CT computerized aided quantification of PNEUMONIA lesions in COVID-19 infection: a comparison among three commercial software. Int J Environ Res Public Health 17

10. Machhi J, Herskovitz J, Senan AM et al (2020) The natural history, pathobiology, and clinical manifestations of SARS-CoV-2 infections. J Neuroimmune Pharmacol 15:359-386

11. Wu J, Wu X, Zeng W et al (2020) Chest CT findings in patients with coronavirus disease 2019 and its relationship with clinical features. Invest Radiol 55:257-261

12. Beltran-Corbellini A, Chico-Garcia JL, Martinez-Poles J et al (2020) Acute-onset smell and taste disorders in the context of 
COVID-19: a pilot multicentre polymerase chain reaction based case-control study. Eur J Neurol 27:1738-1741

13. Carignan A, Valiquette L, Grenier C et al (2020) Anosmia and dysgeusia associated with SARS-CoV-2 infection: an age-matched case-control study. CMAJ 192:E702-E707

14. Levinson R, Elbaz M, Ben-Ami R et al (2020) Time course of anosmia and dysgeusia in patients with mild SARS-CoV-2 infection. Infect Dis (Lond) 52:600-602

15. Vaira LA, Salzano G, Deiana G, De Riu G (2020) Anosmia and ageusia: common findings in COVID-19 patients. Laryngoscope 130:1787

16. Haehner A, Draf J, Drager S et al (2020) Predictive value of sudden olfactory loss in the diagnosis of COVID-19. ORL J Otorhinolaryngol Relat Spec 82:175-180

17. Dell'Era V, Farri F, Garzaro G et al (2020) Smell and taste disorders during COVID-19 outbreak: cross-sectional study on 355 patients. Head Neck 42:1591-1596

18. Speth MM, Singer-Cornelius T, Oberle M et al (2020) Olfactory dysfunction and sinonasal symptomatology in COVID-19: prevalence, severity, timing, and associated characteristics. Otolaryngol Head Neck Surg 163(1):114-120. https://doi.org/10. 1177/0194599820929185. Epub 2020 May 19. PMID: 32423357; PMCID: PMC7240313

19. Vaira LA, Hopkins C, Salzano G et al (2020) Olfactory and gustatory function impairment in COVID-19 patients: Italian objective multicenter-study. Head Neck 42:1560-1569

20. Haehner A, Draf J, Dräger S et al (2020) Predictive value of sudden olfactory loss in the diagnosis of COVID-19. ORL J Otorhinolaryngol Relat Spec 82(4):175-180. https://doi.org/10. 1159/000509143. Epub 2020 Jun 11. PMID: 32526759; PMCID: PMC7360503

21. Han X, Cao Y, Jiang N et al (2020) Novel coronavirus disease 2019 (COVID-19) pneumonia progression course in 17 discharged patients: comparison of clinical and thin-section computed tomography features during recovery. Clin Infect Dis 71:723-731
22. Ye Z, Zhang Y, Wang Y et al (2020) Chest CT manifestations of new coronavirus disease 2019 (COVID-19): a pictorial review. Eur Radiol 30:4381-4389

23. Wang L, He W, Yu X et al (2020) Coronavirus disease 2019 in elderly patients: characteristics and prognostic factors based on 4-week follow-up. J Infect 80:639-645

24. Francone M, Iafrate F, Masci GM et al (2020) Chest CT score in COVID-19 patients: correlation with disease severity and shortterm prognosis. Eur Radiol 30:6808-6817

25. Xiang JWJ, Yuan X et al (2020) Potential biochemical markers to identify severe cases among COVID-19 patients. medRxiv (epub ahead of print)

26. Huang C, Wang Y, Li X et al (2020) Clinical features of patients infected with 2019 novel coronavirus in Wuhan. China Lancet 395:497-506

27. Sanli DET, Altundag A, Kandemirli SG et al (2021) Relationship between disease severity and serum IL-6 levels in COVID-19 anosmia. Am J Otolaryngol 42:102796

28. Chen XLJ, Mo P et al (2020) Restoration of leukomonocyte counts is associated with viral clearance in COVID-19 hospitalized patients. medRxiv (Preprint)

29. Foster KJ, Jauregui E, Tajudeen B et al (2020) Smell loss is a prognostic factor for lower severity of coronavirus disease 2019. Ann Allergy Asthma Immunol 125:481-483

30. Huang YLX, Li D, Wang L et al (2020) A cohort study of 676 patients indicates D-dimer is a critical risk factor for the mortality of COVID-19. PLoS One 15(11): e0242045

31. Zheng YY, Ma YT, Zhang JY, Xie X (2020) COVID-19 and the cardiovascular system. Nat Rev Cardiol 17:259-260

32. Lippi G, Lavie CJ, Sanchis-Gomar F (2020) Cardiac troponin I in patients with coronavirus disease 2019 (COVID-19): evidence from a meta-analysis. Prog Cardiovasc Dis 63:390-391

Publisher's Note Springer Nature remains neutral with regard to jurisdictional claims in published maps and institutional affiliations. 